\title{
ON THE REGULARITY UP TO THE BOUNDARY IN THE DIRICHLET PROBLEM FOR DEGENERATE ELLIPTIC EQUATIONS
}

\author{
ADALBERTO P. BERGAMASCO, JORGE A. GERSZONOWICZ AND \\ GERSON PETRONILHO
}

\begin{abstract}
We give a simple proof of the regularity up to the boundary of solutions of the Dirichlet problem for a class of second-order degenerate elliptic equations in the plane. We show that the method of transfer to the boundary via the associated heat equations, can be used to reduce the problem to proving the ellipticity or hypoellipticity of a pseudodifferential operator on the boundary.
\end{abstract}

\section{INTRODUCTION}

In this article we give a simple proof of the regularity up to the boundary of solutions of the Dirichlet problem for certain second-order degenerate elliptic equations in the plane. We show that the method of transfer to the boundary via the associated heat equations, as introduced by Treves $[6,7]$ in the case of nondegenerate equations, can be used to prove regularity for a class of equations that degenerate at the boundary. The same approach can be applied to (coercive) elliptic boundary value problems other than Dirichlet's.

In $\S 1$ we study regularity up to the boundary for certain pseudodifferential equations, using parametrices of the Cauchy problem for the associated heat equations. This is applied in $\S 2$ to certain factorizable second-order degenerate elliptic differential equations in the plane. We consider two types of degeneracy. In the first one (cf. hypothesis (1.1)) the factors degenerate at a constant rate at the boundary while in the second one (cf. hypothesis $\left.\left(1.1^{\prime}\right)\right)$ they degenerate of high order, possibly at a nonconstant rate or even of infinite order-see the examples given at the end of the paper. In the first case the result about the associated heat equations proved in $\S 1$ was obtained by using Fourier integral operators with complex phase functions whereas in the second case we give an explicit construction-using only pseudodifferential operators-of the needed parametrices; the operator $N$ (cf. Propositions 2 and 3) is elliptic in the first situation but only hypoelliptic in the second one.

Received by the editors December 23, 1987.

1980 Mathematics Subject Classification (1985 Revision). Primary 35J70; Secondary 35S99.

The first author was partially supported by CNPq (Brasil). 


\section{Regularity in the DiRICHLET PROBLEM FOR CERTAIN PSEUDODIFFERENTIAL EQUATIONS}

Let $U \times \Gamma$ be an open conic neighborhood of $\left(x_{0}, \xi_{0}\right) \in R^{n} \times\left(R^{n} \backslash 0\right)$. We shall study regularity for solutions of equations involving pseudodifferential operators $(\psi d o)$ microlocalized to $U \times \Gamma$, depending smoothly on $t \in[0, T]$. In doing so we will eventually shrink $U \times \Gamma$ and $T>0$.

We denote by $\mu\left(t, x, D_{x}\right), \tilde{\mu}\left(t, x, D_{x}\right)$ two classical $\psi d o$ of order $\leq 1$ depending smoothly on $t \in[0, T]$; for simplicity we assume that their total symbols are in $\mathscr{C}^{\infty}\left([0, T] \times U \times R^{n} \backslash 0\right)$. We make either hypothesis (1.1) or hypothesis $\left(1.1^{\prime}\right)$ below.

$$
\begin{array}{ll}
\sigma_{1}(\mu)(t, x, \xi)=t^{k} a(t, x, \xi) \geq 0 & \text { on }[0, T] \times U \times R^{n} \backslash 0, \\
\sigma_{1}(\tilde{\mu})(t, x, \xi)=t^{k} \tilde{a}(t, x, \xi) \geq 0 & \text { on }[0, T] \times U \times R^{n} \backslash 0,
\end{array}
$$

where $k$ is an integer $\geq 0$ and $a, \tilde{a}$ are positive-homogeneous of degree 1 (for $|\xi| \geq 1 / 2$ ), and strictly positive on $[0, T] \times \bar{U}$ for $|\xi|=1, \xi \in \bar{\Gamma}$.

$$
\begin{array}{ll}
\sigma(\mu)(t, x, \xi)=\sigma_{1}(\mu)(t, x, \xi)=t^{k} a(t, x)|\xi| \geq 0 & \text { on }[0, T] \times U \times R^{n} \backslash 0, \\
\sigma(\tilde{\mu})(t, x, \xi)=\sigma_{1}(\tilde{\mu})(t, x, \xi)=t^{k} \tilde{a}(t, x)|\xi| \geq 0 & \text { on }[0, T] \times U \times R^{n} \backslash 0,
\end{array}
$$

where $k$ is an integer $\geq 4$ and $a, \tilde{a}$ are strictly positive on $(0, T] \times \bar{U}$.

Remark. In hypothesis $\left(1.1^{\prime}\right)$, differently from what happens in (1.1), we allow $a, \tilde{a}$ to vanish when $t=0$.

Let

$$
L_{+}=\partial_{t}+\mu\left(t, x, D_{x}\right) \quad \text { and } \quad L_{-}=\partial_{t}-\tilde{\mu}\left(t, x, D_{x}\right) .
$$

In either case, the forward (resp. backward) Cauchy problem for $L_{+}$(resp. $L_{-}$) is well posed and has a parametrix which we denote $G_{+}(t, s)$ (resp. $\left.G_{-}(t, s)\right)$; it is a complex-phase Fourier integral operator depending smoothly on $t \geq s$ (resp. $t \leq s$ ) in [0,T], (if $T$ is small), cf. [5, 4]; under hypothesis $\left(1.1^{\prime}\right)$, we shall construct the parametrices $G_{+}, G_{-}$as pseudodifferential operators (see Lemmas 1, 2, 3).

If we denote by $A \sim 0$ the fact that $A$ is a $\mathscr{C}^{\infty}$ function of $(t, s)$ valued in the space of regularizing operators in the space variables, we have:

$$
\begin{gathered}
L_{+} G_{+}(t, s) \sim 0 \text { for } 0 \leq s \leq t \leq T, \\
\left.G_{+}\right|_{t=s} \sim I ; \\
L_{-} G_{-}(t, s) \sim 0 \text { for } 0 \leq t \leq s \leq T, \\
\left.G_{-}\right|_{t=s} \sim I .
\end{gathered}
$$

Moreover such $G_{ \pm}$are unique modulo $\sim$. The main result of this section is the following regularity result for Dirichlet problems: 
Proposition 1. Let $u \in \mathscr{C}^{\infty}\left([0, T], \mathscr{D}^{\prime}(U)\right)$ satisfy the following:

(a) $W F(u) \subset U \times \Gamma,(0 \leq t \leq T)$;

(b) $\left.u\right|_{t=0}$ is in $\mathscr{C}^{\infty}(U)$;

(c) $u$ satisfies either of the following equations:

(c $\left.c_{1}\right) L_{-} L_{+}(u) \in \mathscr{C}^{\infty}([0, T] \times U)$, or

(c) $L_{+} L_{-}(u) \in \mathscr{C}^{\infty}([0, T] \times U)$.

Then there exists a neighborhood $U^{\prime} \times \Gamma^{\prime} \subset U \times \Gamma$ of $\left(x_{0}, \xi_{0}\right)$ such that $u$ is smooth in $U^{\prime} \times \Gamma^{\prime}$ up to the boundary, i.e. $\psi\left(x, D_{x}\right) u \in \mathscr{C}^{\infty}([0, T] \times U)$ for any proper pseudodifferential operator $\psi\left(x, D_{x}\right)$ whose wave-front is contained in $U^{\prime} \times \Gamma^{\prime}$.

Proof. Shrinking $T$ it is sufficient to show that $\psi\left(x, D_{x}\right) u \in \mathscr{C}^{\infty}([0, T) \times U)$; note that both $L_{-} L_{+}$and $L_{+} L_{-}$are elliptic in $\bar{U} \times \bar{\Gamma}$ for $t>0$, but degenerate at $t=0$ if $k \geq 1$.

We shall consider for simplicity that the smoothness hypothesis holds locally, but it will be clear that since $G_{ \pm}$are microlocal in the space variables $x$, so is the proof. Thus we assume $\Gamma=R^{n} \backslash 0$ from now on.

Case 1. Suppose $u$ satisfies $\left(\mathrm{c}_{1}\right)$. We proceed as in the treatment of regularity in the nondegenerate elliptic case (cf. [6,7]). We uncouple equation $\left(c_{1}\right)$ :

$$
\begin{gathered}
L_{-}(v)=f \in \mathscr{C}^{\infty}, \\
L_{+}(u)=v \text { and }\left.u\right|_{t=0}=u_{0} \in \mathscr{C}^{\infty} .
\end{gathered}
$$

The parametrices $G_{ \pm}$are both regularizing for $t \neq s$ because of the assumption that $\sigma_{1}(\mu)$ and $\sigma_{1}(\tilde{\tilde{\mu}})$ are positive for $t>0$. Using this and the uniqueness of the parametrices $\bmod \sim$, we deduce from (1.4) (denoting by $\approx$ the equivalence modulo smooth functions on $[0, T) \times U)$

$$
v(t, \cdot) \approx G_{-}(t, T) v(T, \cdot)-\int_{t}^{T} G_{-}(t, s) f(s, \cdot) d s
$$

for $0 \leq t \leq T$, and therefore

$$
v \approx 0 \quad \text { (i.e. is smooth for } t<T \text { ). }
$$

Now (1.5) implies

$$
u(t, \cdot) \approx G_{+}(t, 0) u_{0}+\int_{0}^{t} G_{+}(t, s) v(s, \cdot) d s
$$

$(0 \leq t \leq T)$, and by (1.7) and hypothesis (b) this shows that $u$ is $\mathscr{C}^{\infty}$ in $[0, T) \times U$ as desired.

Case 2. Let $u$ satisfy $\left(c_{2}\right)$; this equation uncouples as follows:

$$
\begin{gathered}
L_{+}(v)=f \in \mathscr{C}^{\infty}([0, T] \times U), \\
L_{-}(u)=v \text { and }\left.u\right|_{t=0}=u_{0} \in \mathscr{C}^{\infty}(U) .
\end{gathered}
$$


Denoting by $v_{0}$ the trace $\left.v\right|_{t=0}$, the uniqueness $\bmod \sim$ of $G_{+}$and (1.8) imply

$$
\begin{aligned}
v(t, \cdot) & \approx G_{+}(t, 0) v_{0}+\int_{0}^{t} G_{+}(s, 0) f(s, \cdot) d s \\
& \approx G_{+}(t, 0) v_{0} .
\end{aligned}
$$

Of course this would imply $v \approx 0$ if we knew that $v_{0}$ is smooth. Analogously (1.9) implies

$$
\begin{aligned}
u(t, \cdot) & \approx G_{-}(t, T) u(T, \cdot)-\int_{t}^{T} G_{-}(t, s) v(s, \cdot) d s \\
& \approx-\int_{t}^{T} G_{-}(t, s) v(s, \cdot) d s .
\end{aligned}
$$

Therefore $^{1}$

$$
u(t, \cdot) \approx-\int_{t}^{T} G_{-}(t, s) G_{+}(s, 0) v_{0} d s .
$$

When $t=0$, the initial condition $\left.u\right|_{t=0}=u_{0} \in \mathscr{C}^{\infty}(U)$ shows that

$$
\int_{0}^{T} G_{-}(0, s) G_{+}(s, 0) v_{0}(\cdot) d s
$$

is smooth. Propositions 2 and 3 imply that $\int_{0}^{T} G_{-}(0, s) G_{+}(s, 0) d s$ is a hypoelliptic operator in $U$. This implies that $v_{0}$ is smooth and completes the proof, since then $v \approx 0$ and $u \approx 0$.

Remark. We have $\left.\partial_{t} u\right|_{t=0}=v_{0}+\tilde{\mu}\left(0, x, D_{x}\right) u_{0}$, so if $v_{0}$ is smooth (the Dirichlet boundary value $u_{0}$ being also smooth), then the normal derivative $\left.\partial_{t} u\right|_{t=0}$ will also be smooth. Thus the operator $\int_{0}^{T} G_{-}(0, s) G_{+}(s, 0) d s$ links the smoothness of $\left.u\right|_{t=0}$ and of $\left.\partial_{t} u\right|_{t=0}$ for any solution $u$. This link is classical in the Dirichlet problem for the Laplacian $\Delta=\left(\partial_{t}-i \partial_{x}\right)\left(\partial_{t}+i \partial_{x}\right)$.

Proposition 2. Under hypothesis (1.1), $N\left(x, D_{x}\right)=\int_{0}^{T} G_{-}(0, s) G_{+}(s, 0) d s$ is an elliptic pseudodifferential operator on $U$ of order $-1 /(k+1)$.

Example. When $L_{ \pm}=\partial_{t} \pm t^{k}\left|D_{x}\right|$, the parametrices $G_{ \pm}(t, s)$ are convolution operators with symbols

$$
G_{-}(s, t, \xi)=G_{+}(t, s, \xi)=\exp \left(\left(s^{k+1}-t^{k+1}\right) \frac{|\xi|}{k+1}\right) \quad(t \geq s),
$$

\footnotetext{
${ }^{1}$ Since both $G_{ \pm}(t, s)$ are pseudodifferential for $t=s$, the singular support of their kernels are close to the diagonal for $T$ small $(|t-s| \leq T)$ (in fact, it is contained in the diagonal). Thus is it possible to add regularizing operators to $G_{ \pm}$and obtain proper operators whose composition is well-defined. This will be disregarded below, but in all rigor the amplitudes $K, K_{2}$ in (1.12), (1.13), (1.15) should depend on both $x, y$ to reflect this change. Then $(x, y)$ varies in a small neighborhood of the diagonal; only small changes are needed in the proof of Proposition 2 .
} 
so

$$
\begin{aligned}
N(\xi) & =\int_{0}^{T} \exp \left(-2 s^{k+1} \frac{|\xi|}{k+1}\right) d s \\
& =R(\xi)+\int_{0}^{+\infty} \exp \left(-2 s^{k+1} \frac{|\xi|}{k+1}\right) d s \\
& =R(\xi)+c|\xi|^{-1 /(k+1)}
\end{aligned}
$$

where $c \neq 0$ and $R(\xi)$ is rapidly decreasing for $|\xi| \geq 1$.

Proof of Proposition 2. Recall from [5] that $G_{+}(t, 0)$ is a complex-phase FIO of the form

$$
\left(G_{+}(t, 0) u\right)(x)=(2 \pi)^{-n} \int e^{i \phi(t, x, \xi)} K(t, x, \xi) \hat{u}(\xi) d \xi
$$

for small $T$ and $t \in[0, T]$. The phase $\phi$ is an approximate solution of the eikonal equation $i \phi_{t}+\sigma_{1}(\mu)\left(t, x, \phi_{x}\right)=0,\left.\phi\right|_{t=0}=x \cdot \xi, \sigma_{1}(\mu)$ denoting here and in what follows an almost-analytic extension of the principal symbol of $\mu$. The amplitude $K$, obtained solving approximately the transport equations, is classical and elliptic of order zero.

Denote by $L_{-}^{*}=-\partial_{t}-\tilde{\mu}\left(t, x, D_{x}\right)^{*}$ the $L^{2}$-adjoint of $L_{-}$; the forward Cauchy problem at $t=s$ for $L_{-}^{*}$ has a parametrix $G_{1}(t, s) \quad(t \geq s)$ of the form (1.12) with a phase $\tilde{\phi}$ (solution mod flats of the eikonal equation) and an elliptic zero-order amplitude $K_{1}$, now depending also on $s$. By the uniqueness mod regularizing operators of these parametrices it follows integrating by parts that $G_{-}(t, s) \sim G_{1}(s, t)^{*}(s \geq t)$. The operator $G_{-}(0, s)$ can therefore be written as an oscillatory integral:

$$
\left(G_{-}(0, s) v\right)(x)=(2 \pi)^{-n} \iint e^{i x \cdot \xi-i \overline{\bar{\phi}}(s, y, \xi)} K_{2}(s, y, \xi) v(y) d y d \xi
$$

with $K_{2}$ a classical zero-order elliptic amplitude. From (1.12) and (1.13) we get modulo regularizing operators,

$$
\left[G_{-}(0, s) \circ G_{+}(s, 0) u\right](x)=(2 \pi)^{-n} \int e^{i x \cdot \theta} F(s, x, \theta) \hat{u}(\theta) d \theta
$$

which is a pseudodifferential operator since $F$, defined below, will be shown to belong to $S_{1,0}^{0}$ (uniformly in $s$ ). Interpreting (1.14) and (1.15) as oscillatory integrals we have ${ }^{2}$

$$
\begin{aligned}
F(s, x, \theta)=(2 \pi)^{-n} \iint e^{i[\phi(s, y, \theta)-\bar{\phi}(s, y, \xi)+x \cdot(\xi-\theta)]} & \\
& \times K(s, y, \theta) K_{2}(s, y, \xi) d y d \xi \cdots
\end{aligned}
$$

We estimate the behavior as $|\theta| \rightarrow \infty$ of $F$ by applying the (real) stationary phase theorem to the integral (1.15) after a change of variable $\theta=\tau \omega(|\omega|=1)$, $\xi=\tau \eta$. It suffices to reason microlocally, for $(x, \omega)$ near $\left(x_{0}, \omega_{0}\right)$ and $0 \leq s \leq$

\footnotetext{
2 In (1.15) we can assume that $y$ varies in a compact set; see footnote 1 .
} 
$T$. The real phase $\psi(s, x, \omega, y, \eta)=\operatorname{Re} \phi(s, y, \omega)-\operatorname{Re} \tilde{\phi}(s, y, \eta)+x \cdot(\eta-\omega)$ is equal for $s=0$ to $(y-x) \cdot(\omega-\eta)$, which has a nondegenerate critical point in the variables $(y, \eta)$ at $y=x, \eta=\omega$. Hence $\psi$ has for $s$ small, and $(x, \omega) \in V=$ neighborhood of $\left(x_{0}, \omega_{0}\right)$, a unique nondegenerate critical point depending smoothly on $(s, x, \omega)$, denoted $\gamma(s, x, \omega)$.

We are going to consider the exponential

$$
\exp (-\tau[\operatorname{Im} \phi(s, y, \omega)+\operatorname{Im} \tilde{\phi}(s, y, \eta)])
$$

as part of the amplitude; notice that it is of class $S_{1,0}^{0}\left(V \times V, R_{+}\right)$uniformly in $s$ because by (1.1) and the hypothesis on $a$ and $\tilde{a}$ we have $c t^{k+1}|\xi| \leq$ $\operatorname{Im} \phi(t, x, \xi) \quad(c>0)$ and also $\left|\operatorname{Im} \phi_{x}(t, x, \xi)\right| \leq c t^{k+1}|\xi|,\left|\operatorname{Im} \phi_{\xi}(t, x, \xi)\right| \leq$ $c t^{k+1}$, (and in general $\left|\partial_{x, \xi}^{\alpha}(\operatorname{Im} \phi)\right| \leq c_{\alpha} t^{k+1}$ for $\left.|\xi|=1\right)$ and similar estimates for $\tilde{\phi}$; cf. [5]. Let $J(s, x, \omega, y, \eta, \tau)$ denote the (nonclassical) amplitude in $S_{1,0}^{0}\left(V \times V, R_{+}\right)$depending smoothly on $s \in[0, T]$ :

$$
\chi_{0}(y, \eta) \exp (-\tau[\operatorname{Im} \phi(s, y, \omega)+\operatorname{Im} \tilde{\phi}(s, y, \eta)]) K(s, y, \tau \omega) \cdot K_{2}(s, y, \tau \eta)
$$

where $\chi_{0}$ has compact support in $V$ and is $\equiv 1$ near $\left(x_{0}, \omega_{0}\right), 0 \leq \chi_{0} \leq 1$. Then we have for $(x, \omega)$ near $\left(x_{0}, \omega_{0}\right)$, as $\tau \rightarrow+\infty$,

$$
F(s, x, \tau \omega)=\left(\frac{\tau}{2 \pi}\right)^{n} \iint e^{i \tau \Psi(s, x, \omega, y, \eta)} J(s, x, \omega, y, \eta, \tau) d y d \eta
$$

$\bmod S^{-\infty}$ (uniformly in $0 \leq s \leq T$ ), and by the stationary phase theorem $F$ is in $S_{1,0}^{0}\left(V, R_{+}\right)$uniformly in $s \in[0, T]$, and is equal $\bmod S_{1,0}^{-1}$ to

$$
A(s, x, \omega) k^{0}(s, y, \omega) k_{2}^{0}(s, y, \eta) \exp (-\tau[\operatorname{Im} \phi(s, y, \omega)+\operatorname{Im} \tilde{\phi}(s, y, \eta)]),
$$

where $(y, \eta)=\gamma(s, x, \omega), k^{0}, k_{2}^{0}$ denote the homogeneous principal parts of the classical symbols $K, K_{2}$, respectively, and $A$ is a nonzero factor depending on the Hessian of $\Psi$ at the critical point. Since $k^{0}, k_{2}^{0}$ are elliptic of order zero we can write near $\left(x_{0}, \omega_{0}\right)$ for $0 \leq s \leq T$,

$$
F(s, x, \tau \omega)=B(s, x, \omega) \exp (-\tau[\operatorname{Im} \phi(s, y, \omega)+\operatorname{Im} \tilde{\phi}(s, y, \eta)]) \bmod S_{1,0}^{-1}
$$

(with $(y, \eta)=\gamma(s, x, \omega)$ ), where $|B|$ is bounded above and away from zero.

Moreover, we have $\operatorname{Re} B \geq$ const $>0$ near $\left(x_{0}, \omega_{0}\right), 0 \leq s \leq T$. In effect, by construction (cf. [5]) $k^{0}(0, y, \xi)=1$ and also $k_{2}^{0}(0, y, \xi)=1$ since this is true of $k_{1}^{0}$, the principal part of the amplitude of $G_{1}(t, 0)$ (recall $G_{-}(0, s) \sim$ $\left.G_{1}(s, 0)^{*}\right)$. Therefore $k^{0}(s, y, \omega) k_{2}^{0}(s, y, \eta)$ has real part locally bounded away from zero for $0 \leq s \leq T$ if $T$ is small. On the other hand $A(s, x, \omega)$ is $|\operatorname{det} H(s, x, \omega)|^{-1 / 2} e^{i(\pi / 4) \operatorname{sign} H}$ where $H$ is the Hessian of $\Psi$ at the critical point, and sign $H=0$ since when $s=0, H=\left[\begin{array}{cc}0 & -I \\ -I & 0\end{array}\right]$. Hence $A$ is positive, and locally $\operatorname{Re} B \geq c>0$ for some $c$. 
By the preceding results, the operator $N\left(x, D_{x}\right)=\int_{0}^{T} G_{-}(0, s) G_{+}(s, 0) d s$ is pseudodifferential of order zero (cf. (1.14)), and modulo $S_{1,0}^{-1}$ its symbol is near $\left(x_{0}, \omega_{0}\right)$ equal to

$$
\left.\int_{0}^{T} B(s, x, \omega) \exp [-\tau(\operatorname{Im} \phi(s, y, \omega)+\operatorname{Im} \tilde{\phi}(s, y, \eta))]\right|_{(y, \eta)=\gamma(s, x, \omega)} d s .
$$

To show that (1.17) is in $S_{1,0}^{-1 /(k+1)}\left(R_{+}\right)$near $\left(x_{0}, \omega_{0}\right)$, recall that (cf. [5])

$$
\left|\operatorname{Im} \phi(t, y, \theta)-\int_{0}^{t} \sigma_{1}(\mu)(s, y, \theta) d s\right| \leq \mathrm{const} t \int_{0}^{t} \sigma_{1}(\mu)(s, y, \theta) d s
$$

for $t \in[0, T]$, and by our hypothesis on $\sigma_{1}(\mu)$ we obtain

$$
c_{1} t^{k+1}|\theta| \leq \operatorname{Im} \phi(t, y, \theta) \leq c_{2} t^{k+1}|\theta|
$$

for $t \in[0, T]$ and $(y, \theta /|\theta|)$ near $\left(x_{0}, \omega_{0}\right)$, for some positive constants $c_{1}, c_{2}$ (and similar estimates for $\operatorname{Im} \tilde{\phi}$ ). Since as remarked before $0<c_{1}^{\prime} \leq \operatorname{Re} B \leq c_{2}^{\prime}$, locally the real part of (1.17) can be estimated from above and from below by integrals of the form

$$
c_{1}^{\prime \prime} \int_{0}^{T} \exp \left(-\tau c_{2}^{\prime \prime} s^{k+1}\right) d s \sim c_{3}^{\prime \prime} \tau^{-1 /(k+1)} \quad(\tau \rightarrow+\infty)
$$

with positive $c_{1}^{\prime \prime}, c_{2}^{\prime \prime}, c_{3}^{\prime \prime}$, and the absolute value of (1.17) can be estimated from above by such integrals too. This shows that $N$ is elliptic (in a conic neighborhood of $\left.\left(x_{0}, \omega_{0}\right)\right)$ of the desired order $-1 /(k+1)$ provided $-1 /(k+1)>-1$, i.e. $k \geq 1$. The same conclusion is true if $k=0$, since in fact the remainder in (1.16) is, by the stationary phase theorem, of the form

$$
R=\left.R_{1}(s, x, \omega, \tau) \exp (-\tau[\operatorname{Im} \phi(s, y, \omega)+\operatorname{Im} \tilde{\phi}(s, y, \eta)])\right|_{(y, \eta)=\gamma(s, x, \omega)}
$$

where $R_{1}$ is of order -1 . Hence the symbol of $N\left(x, D_{x}\right)$ differs from (1.17) by $\int_{0}^{T} R(s, x, \omega, \tau) d s$ which, by the same argument used before, is of order $-1-1 /(k+1)=-2$ here. So (1.17) determines the ellipticity of the operator even when $k=0$.

It remains to prove Proposition 3 below which is the analogue, in the case of hypothesis $\left(1.1^{\prime}\right)$, of Proposition 2.

Proposition 3. Under hypothesis $\left(1.1^{\prime}\right)$, the operator

$$
N\left(x, D_{x}\right)=\int_{0}^{T} G_{-}(0, s) G_{+}(s, 0) d s
$$

is a hypoelliptic pseudodifferential operator of order zero on $U$ (with symbol in $\left.S_{1,1 / 2}^{0}(U)\right)$.

We start with the construction of the parametrices $G_{ \pm}$which will be pseudodifferential operators; we will show only how to obtain $G_{+}$, the construction of $G_{-}$being analogous. 
Let

$$
G_{+}(t, s) u(x)=(2 \pi)^{-n} \int e^{i x \cdot \xi} g(t, s, x, \xi) \hat{u}(\xi) d \xi
$$

with

$$
g(t, s, x, \xi)=\sum_{j=0}^{\infty} g_{j}(t, s, x, \xi)
$$

Recall that we wish to solve

$$
\begin{gathered}
L_{+} G_{+}(t, s) \sim 0 ; \quad 0 \leq s \leq t \leq T, \\
\left.G_{+}\right|_{t=s} \sim I
\end{gathered}
$$

where

$$
L_{+}=\partial_{t}+\mu\left(t, x, D_{x}\right) \text { and } \sigma(\mu)=t^{k} a(t, x)|\xi| .
$$

We proceed formally and we have

$$
\begin{aligned}
L_{+} G_{+}(t, s) u(x) & \sim(2 \pi)^{-n} \int e^{i x \cdot \xi}\left(\partial_{t} g+t^{k} a|\xi| g+\sum_{\alpha \neq 0} \frac{1}{\alpha !} \partial_{\xi}^{\alpha} \sigma(\mu) D_{x}^{\alpha} g\right) \hat{u}(\xi) d \xi \\
& \sim(2 \pi)^{-n} \int e^{i x \cdot \xi}\left(\partial_{t} g+t^{k} a|\xi| g+t^{k} a \sum_{\alpha \neq 0} \frac{1}{\alpha !} a_{\alpha}(\xi) D_{x}^{\alpha} g\right) \hat{u}(\xi) d \xi
\end{aligned}
$$

where $a_{\alpha}(\xi)$ is smooth and positive-homogeneous of degree $1-|\alpha|$ with respect to $\xi$.

In order to solve (1.2) it suffices to solve

$$
\begin{gathered}
\partial_{t} g+t^{k} a|\xi| g+t^{k} a \sum_{\alpha \neq 0} \frac{1}{\alpha !} a_{\alpha}(\xi) D_{x}^{\alpha} g \sim 0, \\
g(s, s, x, \xi) \sim 1
\end{gathered}
$$

where $\sim$ now means the equivalence of symbols; it suffices to solve:

$$
\begin{gathered}
\partial_{t} g_{0}+t^{k} a|\xi| g_{0}=0, \quad 0 \leq s \leq t \leq T, \\
\left.g_{0}\right|_{t=s}=1
\end{gathered}
$$

and, for $j=1,2, \ldots$,

$$
\begin{gathered}
\partial_{t} g_{j}+t^{k} a|\xi| g_{j}=-t^{k} a \sum_{\substack{1 \leq|\alpha| \leq\left. j \\
g_{j}\right|_{t=s}}} \frac{1}{\alpha !} a_{\alpha}(\xi) D_{x}^{\alpha} g_{j-|\alpha|}, \quad 0 \leq s \leq t \leq T,
\end{gathered}
$$

The solution of (1.19) is given by

$$
g_{0}(t, s, x, \xi)=\exp \left[-|\xi| \int_{s}^{t} r^{k} a(r, x) d r\right]
$$

while that of (1.20) may be written as

$$
\begin{array}{rl}
g_{j}(t, s, x, \xi)=-\int_{s}^{t} g_{0}(t, r, x, \xi) r^{k} & a(r, x) \\
& \times \sum_{1 \leq|\alpha| \leq j} \frac{1}{\alpha !} a_{\alpha}(\xi) D_{x}^{\alpha} g_{j-|\alpha|}(r, s, x, \xi) d r
\end{array}
$$


We will now define the space of symbols which are relevant to our study.

Let $T>0$ and let $\Omega$ be an open subset of $R^{n}$; set

$$
V_{T}=\left\{(t, s) \in R^{2} ; 0 \leq t \leq s \leq T\right\} .
$$

Let $\lambda(t, x)$ be a smooth nonnegative function.

Definition. A function $b(t, s, x, \xi)$ belongs to the class $A^{m, \lambda}=A^{m, \lambda}(\Omega)$ if $b$ is smooth on $V_{T} \times \Omega \times\left(R^{n} \backslash\{0\}\right)$ and if there exists $B>0$ such that for every $\alpha, \beta \in Z_{+}^{n}$, every $\gamma, \delta \in Z_{+}$and every compact set $K$ contained in $V_{T} \times \Omega$, there exists a constant $c=c(\alpha, \beta, \gamma, \delta, K)$ such that

$$
\begin{aligned}
& \left|\partial_{\xi}^{\alpha} \partial_{x}^{\beta} \partial_{s}^{\gamma} \partial_{t}^{\delta} b(t, s, x, \xi)\right| \\
& \quad \leq C(1+|\xi|)^{m-|\alpha|+\frac{1}{2}|\beta|+\gamma+\delta} \exp \left(-B|\xi| \int_{t}^{s} \lambda(r, x) d r\right)
\end{aligned}
$$

for all $(t, s, x, \xi) \in K \times\left(R^{n} \backslash\{0\}\right)$.

Remark. (i) $\partial_{\xi}^{\alpha} \partial_{x}^{\beta} \partial_{s}^{\gamma} \partial_{t}^{\delta}\left(A^{m, \lambda}\right) \subset A^{m-|\alpha|+\frac{1}{2}|\beta|+\gamma+\delta, \lambda}$,

(ii) $A^{m, \lambda} \subset S_{1,1 / 2}^{m}$, uniformly in $(t, s) \in V_{T}$.

Lemma 1. For each $j=0,1, \ldots, g_{j} \in A^{-j / 2, t^{k} a}(U)$.

The proof is by induction; it uses the remark above and the following

Lemma 2. Let $b \in A^{m, \lambda}(U)$. Then the function

$$
H(t, s, x, \xi)=-\int_{s}^{t} g_{0}(t, r, x, \xi) \lambda(r, x) b(r, s, x, \xi) d r
$$

belongs to $A^{m-1, \lambda}(U)$.

Let $\chi: R \rightarrow R$ be smooth, $0 \leq \chi \leq 1$, with $\chi(t)=0$ if $t \leq 1 / 2$ and $\chi(t)=1$ if $t \geq 1$.

We now choose a sequence $\left(r_{j}\right)$ of positive real numbers so that the function

$$
g^{\#}(t, s, x, \xi)=\sum_{j=0}^{\infty} g_{j}(t, s, x, \xi) \chi\left(\frac{|\xi|}{r_{j}}\right)
$$

belongs to $A^{0, t^{k} a}$; that it is indeed possible to achieve this is the content of the next lemma, whose proof is technical and standard and will be omitted (some other proofs were omitted as well).

Lemma 3. Let $\chi$ be as in the definition of $g^{\#}$. If $\sigma_{j} \in A^{-j / 2, \lambda}(\Omega)$ for $j=$ $0,1, \ldots$, then there exists a sequence $\left(r_{j}\right)$ of positive real numbers such that the function

$$
\sigma(t, s, x, \xi)=\sum_{j=0}^{\infty} \sigma_{j}(t, s, x, \xi) \chi\left(\frac{|\xi|}{r_{j}}\right)
$$


belongs to $A^{0, \lambda}(\Omega)$ and, furthermore, such that for each $N=1,2, \ldots$, the function $\sigma-\sum_{j=0}^{N-1} \sigma_{j}$ is in $A^{-N / 2, \lambda}(\Omega)$.

Define the operator

$$
G_{+}^{\#}(t, s) u(x)=(2 \pi)^{-n} \int e^{i x \cdot \xi} g^{\#}(t, s, x, \xi) \hat{u}(\xi) d \xi .
$$

Since $A^{0, t^{k} a}(U) \subset S_{1,1 / 2}^{0}(U)$ it follows that $G_{+}^{\#}(t, s)$ is a pseudodifferential operator of order zero on $U$, depending smoothly on $t, s$.

Proof of Proposition 3. The first step consists in observing that the operator $N$ is a pseudodifferential operator of order zero. To see this, notice that the operators $G_{-}(0, s)$ and $G_{+}(s, 0)$ are, by construction, pseudodifferential operators of order zero; thus, their composition, as well as the integral with respect to $s$ of the latter are operators of the same type.

The second step is to show that if $K$ is a compact subset of $U$ and if $k \geq 2$, then there exist $C>0$ and $M>0$ such that the symbol of $N$,

$$
\sigma(N)(x, \xi)=\int_{0}^{T} F(s, x, \xi) d s
$$

satisfies

$$
|\sigma(N)(x, \xi)| \geq C|\xi|^{-1 /(k+1)}
$$

for $(x, \xi) \in K \times\left(R^{n} \backslash\{0\}\right),|\xi| \geq M$.

In order to prove this we start by observing that the operators which are solutions of (1.2) and of (1.3), have symbols equivalent to $g(t, s, x, \xi)$ and, say, $h(t, s, x, \xi)$, respectively.

The principal symbols are

$$
g_{0}(t, s, x, \xi)=\exp \left[-|\xi| \int_{s}^{t} r^{k} a(r, x) d r\right], \quad 0 \leq s \leq t \leq T,
$$

and

$$
h_{0}(t, s, x, \xi)=\exp \left[-|\xi| \int_{t}^{s} r^{k} \tilde{a}(r, x) d r\right], \quad 0 \leq t \leq s \leq T .
$$

The symbol $F(t, x, \xi)$ is such that

$$
F-\sum_{|\alpha| \leq N-1} \frac{1}{\alpha !} \partial_{\xi}^{\alpha} h D_{x}^{\alpha} g \text { is in } S_{1,1 / 2}^{-N / 2}
$$


We now show that (1.21) holds.

$$
\begin{aligned}
|\sigma(N)(x, \xi)|=\left|\int_{0}^{T} F(s, x, \xi) d s\right| \geq\left|\int_{0}^{T} h_{0}(0, s, x, \xi) g_{0}(s, 0, x, \xi) d s\right| \\
\quad-\int_{0}^{T}\left|h(0, s, x, \xi) g(s, 0, x, \xi)-h_{0}(0, s, x, \xi) g_{0}(s, 0, x, \xi)\right| d s \\
\quad-\int_{0}^{T}|F(s, x, \xi)-h(0, s, x, \xi) g(s, 0, x, \xi)| d s \\
\geq\left|\int_{0}^{T} \exp \left(-|\xi| \int_{0}^{s} r^{k}[a(r, x)+\tilde{a}(r, x)] d r\right) d s\right|-C_{1}|\xi|^{-1 / 2}-C_{2}|\xi|^{-1 / 2} \\
\geq \int_{0}^{T} \exp \left(-C_{0}|\xi| s^{k+1}\right) d s-C_{3}|\xi|^{-1 / 2} \\
\geq C_{4}|\xi|^{-1 /(k+1)}-C_{3}|\xi|^{-1 / 2} \\
\geq C|\xi|^{-1 /(k+1)},
\end{aligned}
$$

provided $|\xi|$ is sufficiently large; $C_{0}>0$.

Notice that the hypothesis $k \geq 2$ was used only once, namely in the last inequality.

For the final step, let $\mathscr{O}$ be a relatively compact open subset of $U$ and suppose $k \geq 4$. Set $m=1 /(k+1), \rho=1-1 /(k+1), \delta=1 / 2+1 /(k+1)$. Then we claim that there exists a pseudodifferential operator $E$, with symbol in $S_{\rho \delta}^{m}(U)$ such that $E N-I$ is regularizing in $\mathscr{O}$. Indeed, $k \geq 4$ implies $\rho>\delta$ and thus, by taking (1.21) into account, it is possible to construct explicitly, in a standard way such a parametrix $E$.

\section{APPLICATION TO SECOND-ORDER DEGENERATE ELLIPTIC DIFFERENTIAL EQUATIONS}

We can now apply Proposition 1 to obtain a regularity result for solutions of the Dirichlet problem for certain degenerate elliptic equations in the plane.

Theorem 3. Let $k \geq 0$ be an integer, $T>0$, and $U \subset R$ a neighborhood of the origin. Let $a, \tilde{a}, b, \tilde{b}$ be smooth functions on $[0, T] \times \bar{U}$. We assume $a$ and $\tilde{a}$ are real, and a ã $<0$. Let

$$
P=\left(\partial_{t}+i t^{k} a(t, x) \partial_{x}+b(t, x)\right)\left(\partial_{t}+i t^{k} \tilde{a}(t, x) \partial_{x}+\tilde{b}(t, x)\right) .
$$

If $u \in \mathscr{C}^{\infty}\left([0, T], \mathscr{D}^{\prime}(U)\right)$ satisfies

$$
\begin{gathered}
P u=f \in \mathscr{C}^{\infty}([0, T] \times U), \\
\left.u\right|_{t=0}=u_{0} \in \mathscr{C}^{\infty}(U),
\end{gathered}
$$

then $u \in \mathscr{C}^{\infty}([0, T] \times U)$, perhaps after shrinking $T, U$.

Proof. Let $\chi(\xi)$ be a smooth function on $R$ that coincides with Heaviside's function for $\xi \leq 0$ and $\xi \geq 1$. We will show that $\chi\left( \pm D_{x}\right) u$ are both in 
$\mathscr{C}^{\infty}([0, T] \times U)$. We have $P\left(\chi\left(D_{x}\right) u\right)+P\left(\chi\left(-D_{x}\right) u\right)=f+$ smooth, and $W F\left(P\left(\chi\left( \pm D_{x}\right) u\right)\right) \subset\{t=0$ and $\pm \xi>0\}$, so both terms $P\left(\chi\left( \pm D_{x}\right) u\right)$ are smooth; also both traces $\left.\chi\left( \pm D_{x}\right) u\right|_{t=0}$ must be smooth.

It suffices now to apply Proposiion 1 (with $n=1$ ) to the cone $\Gamma=\{\xi>0\}$ in the case of $\chi\left(D_{x}\right) u$, and to $\Gamma=\{\xi<0\}$ in the case of $\chi\left(-D_{x}\right) u$, with the obvious choices of $\mu$ and $\tilde{\mu}$. For example, if we assume $a>0, \tilde{a}<0$, then we choose

$$
\begin{aligned}
& L_{-}=\partial_{t}-t^{k} a(t, x)\left|D_{x}\right|+b(t, x), \\
& L_{+}=\partial_{t}-t^{k} \tilde{a}(t, x)\left|D_{x}\right|+\tilde{b}(t, x)
\end{aligned}
$$

in the cone $\{\xi>0\}$, while in the case of the cone $\{\xi<0\}$ the choice is

$$
\begin{aligned}
& L_{-}=\partial_{t}+t^{k} \tilde{a}(t, x)\left|D_{x}\right|+\tilde{b}(t, x), \\
& L_{+}=\partial_{t}+t^{k} a(t, x)\left|D_{x}\right|+b(t, x) .
\end{aligned}
$$

The proof above is also a proof of

Theorem 4. Let $k \geq 4$ be an integer, $T>0$, and $U \subset R$ a neighborhood of the origin. Let $a, \tilde{a}$ be smooth functions on $[0, T] \times \bar{U}$. We assume $a$ and $\tilde{a}$ are real and a a $<0$ when $t>0$. Let $P=\left(\partial_{t}+i t^{k} a(t, x) \partial_{x}\right)\left(\partial_{t}+i t^{k} \tilde{a}(t, x) \partial_{x}\right)$. If $u \in \mathscr{C}^{\infty}\left([0, T], \mathscr{D}^{\prime}(U)\right)$ satisfies

$$
P u=f \in \mathscr{C}^{\infty}([0, T] \times U),\left.\quad u\right|_{t=0}=u_{0} \in \mathscr{C}^{\infty}(U),
$$

then $u \in \mathscr{C}^{\infty}([0, T] \times U)$, perhaps after shrinking $T, U$.

Remark 1. By comparing the hypotheses of Theorems 3 and 4 one notices that in the latter we assume $k \geq 4$ but, on the other hand, we do not require $a, \tilde{a}$ to be nonzero at $t=0$; this allows us to handle operators such as the ones in Examples (i) and (ii) below. Example (iii) is contained in Theorem 3 but not in Theorem 4.

Examples. (i) $P=\left(\partial_{t}+i \phi(t) \partial_{x}\right)\left(\partial_{t}+i \Psi(t) \partial_{x}\right)$, with $\phi, \Psi$ real, flat at $t=0$ and $\phi \Psi<0$ when $t>0$.

(ii) $P=\left(\partial_{t}+i t^{4}\left(t^{2}+x^{2}\right) \partial_{x}\right)\left(\partial_{t}-i t^{4}\left(t^{2}+x^{2}\right) \partial_{x}\right)$.

(iii) $P=\left(\partial_{t}+i t^{2} \partial_{x}\right)\left(\partial_{t}-i t^{2} \partial_{x}\right)$.

Remark 2. When $a=-\tilde{a}$ the proof of Theorem 3 can be simplified; in fact, a change of the space variable, and the fact that $\partial_{t} \pm i t^{k} \partial_{y}$ are locally solvable (in $\mathscr{C}^{\infty}$ ) for $t \geq 0$, allows us to reduce the problem to the case when $P=$ $\left(\partial_{t}+i t^{k} \partial_{y}\right) \cdot c(t, y) \cdot\left(\partial_{t}-i t^{k} \partial_{y}\right)$, with $c \neq 0$ smooth. In this case the parametrices used in Proposition 1 are simpler (cf. example following Proposition 2).

Remark 3. A result analogous to Theorem 3 can be proved for operators similar to $P$ but with principal part $\left(\partial_{t}+i t^{k} a(t, x) \partial_{x}\right) \cdot\left(\partial_{t}+i t^{l} \tilde{a}(t, x) \partial_{x}\right)$ where the nonnegative integers $k, l$ may be different now. It suffices to use a variant of Proposition 1 ; the operator $N\left(x, D_{x}\right)$ will be elliptic of order $-[1+\min (k, l)]^{-1}$ now. 
Remark 4. Theorem 4 applies to operators of the form

$$
\left(\partial_{t}+i t^{k} a(t, x) \partial_{x}\right)\left(\partial_{t}+i t^{l} \tilde{a}(t, x) \partial_{x}\right)
$$

if $l, k \geq 4$; indeed if, e.g., $l \geq k \geq 4$ then we may write $t^{l} \tilde{a}=t^{k}\left(t^{l-k} \tilde{a}\right)$.

\section{REFERENCES}

1. M. S. Baouendi, Sur une classe d'opérateurs elliptiques dégénérés, Bull. Soc. Math. France 95 (1967), 45.

2. V. V. Grusin and M. I. Visik, Boundary value problems for elliptic equations degenerate on the boundary of a domain, Math. USSR-Sb. 9 (1969), 423.

3. L. Hörmander, Pseudo-differential operators and hypoelliptic equations, Proc. Sympos. Pure Math., vol. 10, Amer. Math. Soc., Providence, R. I., 1966, pp. 138-183.

4. J. Sjöstrand, Fourier integral operators with complex phase functions, Seminar on Singularities of Solutions of Linear Partial Differential Equations, Ann. of Math. Studies, no. 91, Princeton Univ. Press, 1979.

5. F. Treves, Solution of Cauchy problems modulo flat functions, Comm. Partial Differential Equations 1 (1976), 45.

6. __ Boundary value problems for elliptic equations, Lecture notes, Bressanone, June 1977.

7. __ Introduction to pseudo-differential and Fourier integral operators (2 vols.), Plenum, New York, 1980.

Departamento de Matemática, Universidade Federal de São Carlos, 13.560 São CarLOS, SP, BRASIL (Current address of A. P. Bergamasco and Gerson Petronilho)

Department of Mathematics, University of Connecticut, Groton, Connecticut 06340 (Current address of J. A. Gerszonowicz) 